\title{
I. Beiträge zur optischen Kenntnis der Hornblenden.
}

\author{
Von \\ W. E. Ford in New Haven (Conn.). \\ (Übersetzt von K. Spangenberg.)
}

(Mit 11 Textfiguren.)

Der verstorbene Professor S. L. Penfield war kurz vor seinem Tode noch mit einer Untersuchung über die chemische Zusammensetzung der Mineralien der Hornblendegruppe beschäftigt. Unter seiner Leitung war zu diesem Zwecke von Dr. F. C. Stanley eine Reihe von elf Amphibolanalysen gemacht worden, die in der Zusammensetzung von Tremolit bis zur gemeinen Hornblende wechselten. Die zur Analyse ausgewählten Exemplare gewährleisteten ein vortreffliches Material zur chemischen Untersuchung, und sicherlich existieren nur wenig ebenso exakte Analysen der Hornblendemineralien. In bezug auf die Diskussion der chemischen Zusammensetzung dieser Amphibole war die Abhandlung zur Zeit von Professor Penfield's Tod praktisch vollendet und wurde daher damals veröffentlicht 1). Die Absicht Penfield's war, diese Arbeit über die chemische Zusammensetzung durch eine Untersuchung der optischen Eigenschaften der gleichen Hornblenden zu ergänzen. Leider konnte er diesen Plan nicht mehr ausführen; es schien mir aber außerordentlich wichtig, diese Arbeit noch zu vollenden. In den meisten Fällen waren die nämlichen Stücke, von denen das Analysenmaterial genommen worden war, noch in der Brush'schen Sammlung aufbewahrt. In den wenigen Fällen, wo es unmöglich war, das Exemplar, das das Material geliefert hatte, einwandfrei zu identifizieren, wurde ein unzweifelhaft aus der gleichen Suite stammendes Stück der gleichen Sammlung benutzt.

1) S. L. Penfield und F. C. Stanley, diese Zeitschr. (1907), 43, 233-260. Amer. Journ. of Sc. (1907), 23. 
Bei Durchsicht der Literatur der letzten 25 Jahre ist man überrascht darüber, in wie wenig Fällen sowobl Analyse wie optische Beschreibung der gleichen Hornblende ausgeführt worden ist. Bei einer ganzen Reihe von Beispielen ist entweder die eine oder die andere angegeben, aber leider finden sich nur selten beide zusammen in der gleichen Beschreibung. Daher schien es doch angebracht, selbst wenn es sich nur um eine einfache Registrierung von Tatsachen handeln sollte, die Untersuchung dieser Hornblenden durch ihre optische Bestimmung zu vervollständigen. Diese Arbeit wurde vor einer Reihe von Jahren im Laboratorium von Professor Rosenbusch in Heidelberg begonnen, konnte aber aus verschiedenen Gründen erst kürzlich zum Abschluß gebracht werden.

Soweit als möglich wurden in jedem einzelnen Falle die folgenden Bestimmungen ausgeführt: 1. Brechungsindices und deren Mittelwert, 2. Auslöschungsschiefe auf $b(010)$ gemessen gegen die Richtung der prismatischen Spaltbarkeit und 3. Pleochroismus. Der Wert für den optischen Axenwinkel $2 \mathrm{~V}$ wurde, wo es möglich war, berechnet, direkte Messungen wurden nicht ausgeführt. Die Brechungsindices wurden am Totalrefraktometer gemessen, und zwar wurden in jedem Falle wenigstens zwei verschieden orientierte Schnitte verwendet. In wenigen Fällen, wo die Beschaffenheit des Materials die Anwendung des Totalrefraktometers unmöglich machte, wurde der Mittelwert der Brechungsindices unter dem Mikroskop durch Immersion kleiner Bruchstücke in Flüssigkeiten von bekanntem Brechungsindex bestimmt. Die Auslöschungsschiefe von $b(010)$ wurde an sorgfältig orientierten Schnilten bei Anwendung von $\mathrm{Na}$-Licht bestimmt; um einen guten Mittelwert zu erhalten, wurden die Ablesungen müglichst oft wiederbolt. Meist waren diese Schliffe von gut ausgebildeten Krystallen gewonnen, sodaß die Neigungsrichtung der $a$-Axe bekannt war. In allen diesen Fällen wurde die Auslöschungsrichtung $\mathfrak{c}$, wie gewöhnlich bei Amphibolen, im stumpfen Winkel $\beta$ liegend gefunden. Bei den pleochroitischen Varietäten wurde ein zweiter Schnitt parallel $a(100)$ hergestellt, um die Schwingungsrichtung $\mathfrak{b}$ zu erhalten. Die so gewonnenen Resultate sind im folgenden kurz zusammengestellt, die entsprechenden Analysen sind in der Tabelle auf S. 4 enthalten.

1. Tremolit von Richville bei Gouverneur, New York. $\alpha=1,5992, \beta=1,6132, \gamma=1,6246$; Mittelwert d. Br. $=1,612$. $\gamma-\alpha=0,0254$.

$2 V=83^{\circ} 23^{\prime} . \quad \mathfrak{c} \sim c=20^{\circ} 1^{\prime}$.

2. Tremolit von Lee, Massachusetts.

$\alpha=1,6022, \beta=1,6192, \gamma=1,6347$; Mittelwert d. Br. $=1,618$. $\gamma-\alpha=0,0325$.

$2 V=86^{\circ} 29^{\prime}$. c $\sim c^{\prime}=16^{\circ} 38^{\prime}$. 
3. Aktinolith von Russell, St. Lawrence Co., New York.

$\alpha=1,6162, \beta=1,6304, \gamma=1,6412$; Mittelwert d. $\mathrm{Br} .=1,629$. $\gamma-\alpha=0,0250$.

$2 V=81^{\circ} 30^{\prime} . \quad \mathrm{c} \wedge \mathrm{c}=14^{\circ} 47^{\prime}$.

4. Aktinolith vom Greiner in Tirol.

$\alpha=1,6173, \beta=1,6330, \gamma=1,6412 ;$ Mittelwert d. $\mathrm{Br} .=1,631$. $\gamma-\alpha=0,0277$.

$2 V=81^{\circ} 38^{\prime} . \quad \mathrm{c} \wedge \stackrel{c}{c}=14^{\circ} 59^{\prime}$.

5. Aktinolith von Pierrepont, St. Lawrence Co., New York.

$\alpha=1,6237, \beta=1,6382, \gamma=1,6503$; Miltelwert d. Br. $=1,637$. $\gamma-\alpha=0,0236$.

$2 V=84^{\circ} 8^{\prime}$. c $\curvearrowright \stackrel{e}{e}$ konnte wegen undulöser Auslöschung nicht gemessen werden.

Pleochroismus: $\mathfrak{a}=$ fast farblos; $\mathfrak{b}=$ schwach gelblichgrün; $\mathfrak{c}=$ schwach bläulichgrün.

Absorption: $\mathfrak{c}>\mathfrak{b}>\mathfrak{a}$.

6. Aktinolith aus den Minen von Kragerö, Norwegen.

$\alpha=1,6280, \beta=1,6442, \gamma=1,6547$; Miltelwert d. Br. $=1,641$. $\gamma-\alpha=0,0267$.

$2 V=76^{\circ} 58^{\prime} . \quad c \sim c=13^{\circ} 35^{\prime}$.

Pleochroismus: $\mathfrak{a}$ und $\mathfrak{b}=$ blaßbraun; $\mathfrak{c}=$ hellgrün.

Absorption: $\mathfrak{c}>\mathfrak{b}=\mathfrak{a}$.

7. Hornblende von Edenville, Orange Co., New York.

$\alpha=1,6583, \beta=1,6701, \gamma=1,6789$; Mittelwert d. Br. $=1,668$. $\gamma-\alpha=0,0206$.

$2 V=81^{\circ} 42^{\prime} . \quad c>c=23^{\circ} 48^{\prime}$.

Pleochroismus: $\mathfrak{a}$ und $\mathfrak{b}=$ lichtbräunlichgrün; $\mathfrak{c}=$ dunkelgrūn. Absorption: $\mathfrak{c}>\mathfrak{b}=\mathfrak{a}$.

8. Hornblende von Renfrew, Ontario, Canada.

Miltelwert d. Br. $=1,67$. c $\frown \stackrel{c}{c}=33^{\circ} 20^{\prime}$.

Pleochroismus: $\mathfrak{a}$ und $\mathfrak{b}=$ olivengrün; $\mathfrak{c}=$ dunkelblaugrün.

Absorption: $\mathfrak{c}>\mathfrak{b}>\mathfrak{a}$.

9. Hornblende von der Mte. Somma, Italien.

Miltelwert d. Br. $=1,68$.

Pleochroismus: $\mathfrak{a}$ und $\mathfrak{b}=$ olivengrün; $\mathfrak{c}=$ dunkelblaugrün.

Absorption: $\mathfrak{c}>\mathfrak{b}=\mathfrak{a}$.

10. Hornblende von Cornwall, Orange Co., New York.

Mittelwert d. Br. $=1,71 . \quad \mathrm{c} \wedge \stackrel{\mathrm{c}}{\mathrm{c}}=9^{\circ}$.

Pleochroismus: $\mathfrak{a}=$ hellolivengrün; $\mathfrak{b}=$ gelblichgrün, $\mathfrak{c}=$ bläulichgrün.

Absorption: $\mathfrak{c}>\mathfrak{b}>\mathfrak{a}$. 
W. E. Ford.

\begin{tabular}{|c|c|c|c|c|c|c|c|c|c|c|}
\hline $\mathrm{SiO}_{2}$ & $\begin{array}{c}1 . \\
57,45\end{array}$ & $\begin{array}{c}2 . \\
57,69\end{array}$ & $\begin{array}{c}3 . \\
54,80\end{array}$ & $\begin{array}{c}4 . \\
56,25\end{array}$ & $\begin{array}{c}5 . \\
52,31\end{array}$ & $\begin{array}{c}6 . \\
51,85\end{array}$ & $\begin{array}{c}7 . \\
41,99\end{array}$ & $\begin{array}{c}8 . \\
43,76\end{array}$ & $\begin{array}{c}9 . \\
39,48\end{array}$ & $\begin{array}{c}10 . \\
36,86\end{array}$ \\
\hline $\mathrm{TiO}_{2}$ & - & 0,14 & 0,10 & - & 0,28 & 1,26 & 1,46 & 0,78 & 0,30 & 1,04 \\
\hline $\mathrm{Al}_{2} \mathrm{O}_{3}$ & 1,30 & 1,80 & 2,58 & 1,24 & 2,69 & 4,36 & 11,62 & $8,33^{\circ}$ & 12,99 & 12,10 \\
\hline $\mathrm{Fe}_{2} \mathrm{O}_{3}$ & 0,18 & - & 2,50 & 0,78 & 3,09 & 2,58 & 2,67 & 6,90 & 7,25 & 7,41 \\
\hline $\mathrm{FeO}$ & 0,22 & 0,55 & 4,75 & 5,50 & 6,68 & 5,46 & 14,32 & 10,47 & 10,73 & 23,35 \\
\hline $\mathrm{MnO}$ & 0,07 & Sp. & Sp. & 0,48 & 0,70 & 0,35 & 0,25 & 0,50 & 1,00 & 0,77 \\
\hline $\mathrm{MgO}$ & 24,85 & $24 ; 12$ & 20,30 & 21,19 & 19,27 & 19,48 & 11,17 & 12,63 & 11,47 & 1,90 \\
\hline $\mathrm{CaO}$ & 12,89 & 13,19 & 12,08 & 12,08 & 11,88 & 10,60 & 11,52 & 9,84 & 12,01 & 10,59 \\
\hline $\mathrm{K}_{2} \mathrm{O}$ & 0,54 & 0,22 & 0,24 & 0,28 & 0,50 & 0,35 & 0,98 & 1,28 & 2,39 & 3,20 \\
\hline $\mathrm{Na}_{2} \mathrm{O}$ & 0,67 & 0,48 & 0,82 & 0,19 & 0,78 & 2,15 & 2,49 & 3,43 & 1,70 & 1,20 \\
\hline $\mathrm{H}_{2} \mathrm{O}$ & 1,16 & 1,56 & 1,60 & 1,81 & 1,42 & 1,21 & 0,61 & 0,65 & 0,76 & 1,30 \\
\hline$F_{2}$ & 0,77 & 0,37 & 0,77 & 0,04 & 0,93 & 0,46 & 0,80 & 1,82 & 0,05 & 0,27 \\
\hline \multirow[t]{2}{*}{ Verl. bei $110^{\circ}$} & 0,09 & 0,10 & 0,11 & - & 0,08 & 0,13 & 0,08 & 0,10 & 0,19 & - \\
\hline & 100,19 & 100,22 & 100,65 & 99,84 & 100,59 & 100,24 & 99,96 & 100,49 & 100,25 & 99,99 \\
\hline \multirow[t]{2}{*}{$O=F_{2}$} & 0,32 & 0,15 & 0,32 & - & 0,39 & 0,22 & 0,33 & 0,76 & 0,02 & 0,11 \\
\hline & 99,87 & 00,07 & 100,33 & - & 100,20 & 100,02 & 99,63 & 99,73 & 100,23 & 99,88 \\
\hline
\end{tabular}

Analyse Nr. 1-9 von F. C. Stanley, Nr. 10 von J. L. Nelson.

Mit Hilfe der oben wiedergegebenen Tatsachen kann man über die optischen Eigenschaften von solchen Amphibolen, die wir normal und typisch nennen wollen, folgende allgemeine Angaben machen: Der Mittelwert der Brechungsindices liegt ungefähr $z$ wischen 1,61 und 1,71; für typischen Tremolit liegt er zwischen 1,61 und 1,62, für typischen Aktinolith zwischen 1,63 und 1,64 und für typische Hornblende zwischen 1,66 und 1,71. Die Doppelbrechung ist in der ganzen Serie nahezu gleich stark; die Grenzwerte sind 0,0206 in der Hornblende von Edenville ( $\mathrm{Nr}$. 7) und 0,0325 in dem Tremolit von Lee (Nr. 2), der Durchschnittswert für die ganze Reihe ist 0,0259 . Soweit sie berechnet werden konnten, lagen die Axenwinkel zwischen etwa $77^{\circ}$ und $86^{\circ} 30^{\prime}$. Die Auslöschungsschiefe zwischen $c$ und $c^{\prime}$ gemessen auf $b(010)$ schwankte zwischen $16^{\circ}$ und $20^{\circ}$ bei Tremolit, zwischen $13^{\circ} 30^{\prime}$ und $15^{\circ}$ bei Aktinolith und zwischen $9^{\circ}$ und über $33^{\circ}$ bei den typischen Hornblenden. Starker Pleochroismus fand sich nur bei den Hornblenden, die Aktinolithglieder der Reihe zeigten, wenn überhaupt, nur schwachen Pleochroismus. Die Absorption war stets $\mathfrak{c}>\mathfrak{b}>\mathfrak{a}$, obwohl häufig nur geringe Unterschiede in der Farbe des parallel $\mathfrak{a}$ und $\mathfrak{b}$ schwingenden Lichtes unterschieden werden konnten. Die optische Axenebene war immer die Symmetrieebene, und die Bisektrix $c$ lag im stumpfen Winkel $\beta$. Der Charakter der Doppelbrechung wurde, soweil er geprüft werden konnte, stets negativ gefunden.

Bei unserer gegenwärtigen dürftigen Kenntnis über die Beziehungen zwischen chemischer Zusammensetzung und optischen Eigenschaften sind solche allgemeine Angaben, wie sie oben gemacht wurden, vielleicht alles, was erreichbar sein kann. Die Amphibole bieten wegen der mannigfach wechselnden Zusammensetzung und der großen Zahl variabler Elemente, 
die sie enthalten, einem exakten Vergleich zwischen chemischen und optischen Eigenschaften außerordentliche Schwierigkeiten dar. Es ist fast möglich, $\mathrm{da} ß$ eine derartige Beziehung überhaupt niemals aufgestellt werden kann. Dennoch glaube ich, daß innerhalb einigermaßen weiter Grenzen und unter Anerkennung zahlreicher Ausnahmen versuchsweise auf der Grundlage der an dieser Reihe durchgeführten Untersuchungen Beziehungen angegeben werden können.

Bei Betrachtung der obigen optischen Beziehungen mit Rücksicht auf die entsprechenden $A$ nalysen fand sich, da $B$ vor jeder anderen optischen Eigenschaft die Änderung des Mittelwertes der Brechungsindices geeignet war, Beziehungen zur Änderung der chemischen Zusammensetzung zu zeigen. Daher sind die Analysen hauptsüchlich mit dem Bestreben durchgearbeitet worden, wenn möglich die Art ihrer Änderung bei zunehmendem Mittelwert des Brechungsvermögens festzustellen. So wie die Analysen auf S. 4 angegeben sind, sind sie nach steigendem Betrag dieses Mittelwertes angeordnet. Um die Beziehungen deutlicher zu machen, wurden sie in Fig. 1-9 graphisch dargestellt. Dort sind die Mittelwerte der Brechungsindices als Abszissen, die Prozente der verschiedenen in den Mineralien enthaltenen Elemente als Ordinaten eingetragen. Die Nummern der verschiedenen Punkte entsprechen denen der Analysen auf S. 4. In jeder Figur wurde eine Gerade gezogen, die so dicht als möglich bei den verschiedenen Punkten liegt. Höchstwahrscheinlich würde eine Kurve die Beziehungen zwischen den Brechungsindices und der Zusammensetzung richtiger ausgedrückt haben, aher es schien bei den verfügbaren Daten besser, diese Kurve als Gerade anzugeben. Es muß noch bemerkt werden, daß die ersten sechs Analysen eine Reihe bilden, deren Glieder dicht nebeneinander liegen. Dann kommt eine ziemliche Lücke bis zu Analyse 7-9, die wieder eine Gruppe bilden. Dann kommt wieder ein Sprung und am Ende liegt allein Analyse 10. Leider standen andere Daten, durch die diese Lücken hätten geschlossen werden künnen, nicht zur Verfügung.

Fig. 1 zeigt die $\mathrm{Abnahme} \mathrm{der} \mathrm{SiO}_{2}$-Prozente bei zunehmendem Mittelwert des Brechungsvermügens. Hier wird die Beziehung praktisch recht gut durch die in der Figur angegebene Gerade ausgedrückt. Von den ersten neưn Analysen weicht $\mathrm{Nr}$. 4 am weitesten von dieser theoretischen Linie $a b$ und zwar um einen Betrag von ungefähr 2,5\%. $\Lambda$ us Fig. 2 ist der Zunahme des Mittelwertes der Brechungsindices entsprechend die Zunahme des $\mathrm{Al}_{2} \mathrm{O}_{3}$-Gehaltes zu ersehen. Die für diesen Fall bestimmten Punkte lassen zwar allgemein eine größere Abweichung von der Geraden erkennen als bei der Kieselsäure, doch beträgt hier die größte $\Lambda$ bweichung bei den neun ersten Analysen nur wenig über $2 \%$. Fig. 3, für die Änderung im $\mathrm{Fe}_{2} \mathrm{O}_{3}$-Gehalt, zeigt fast das Gleiche; die größte Abweichung beträgt etwa 2,7\% bei Analyse 7. Fig. 4 enthält die Veränderungen in 
bezug auf die Summe von $\mathrm{Al}_{2} \mathrm{O}_{3}+\mathrm{Fe}_{2} \mathrm{O}_{3}$. Wie zu erwarten, nähern sich in diesem Falle die Punkte im allgemeinen viel mehr der Geraden als in den beiden vorangegangenen, und nur Analyse 4, 9 und 10 weichen etwas merklicher davon ab. Fig. 5 enthält die Änderung des $\mathrm{FeO}$-Gehaltes. Acht

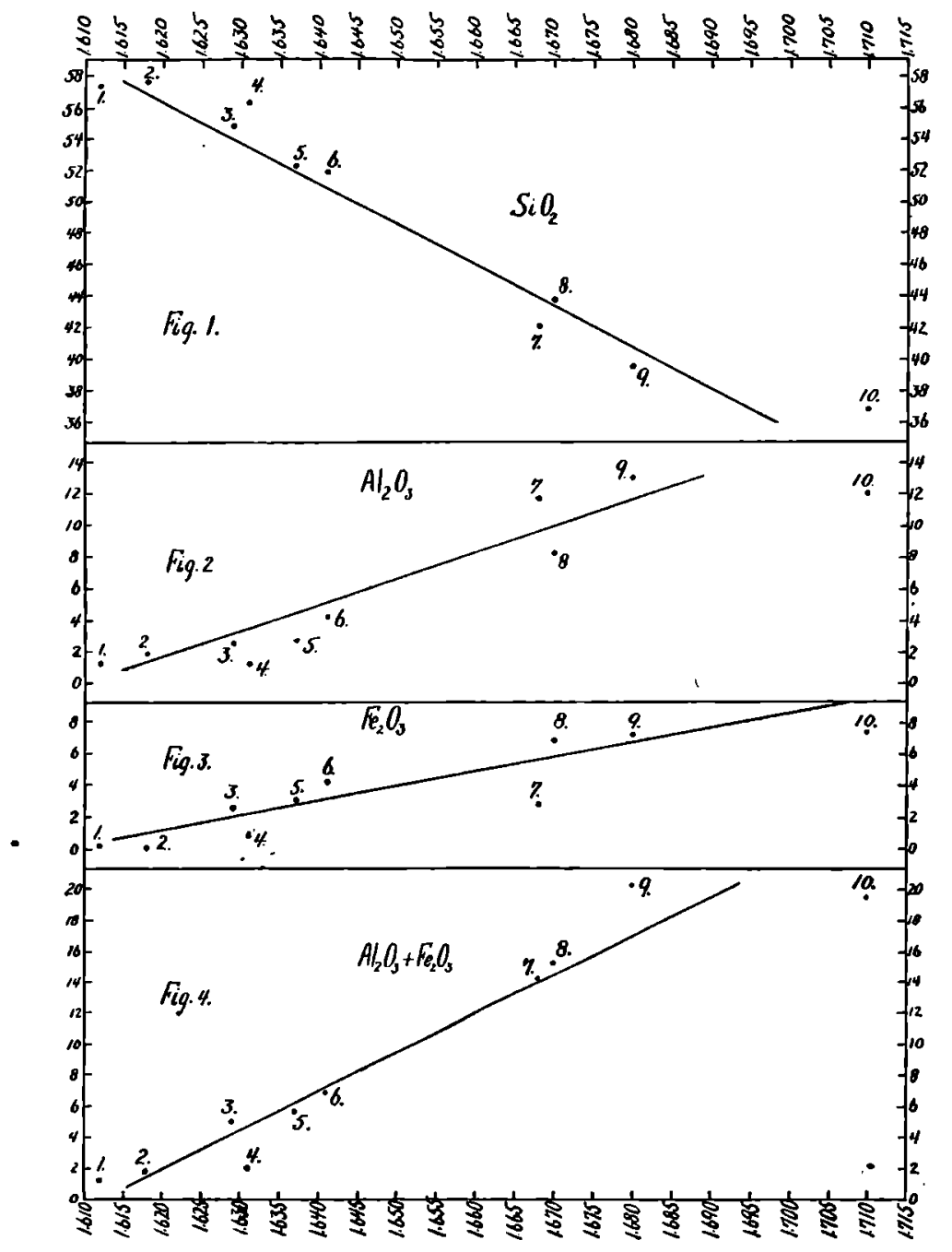

Analysen liefern hier Punkte, die genügend dicht bei der Geraden liegen; dagegen liegen die Analysenpunkte 8 und 9 beträchtlich weit dovon ab, bei 9 beträgt die Abweichung nahezu $6 \%$. Ganz verschieden davon ist die aus Fig. 6 ersichtliche Übereinstimmung in bezug auf die Gesamtsumme des Eisens. Hier ist Analyse 9 die einzige, die eine merkliche, aber auch nur etwa $2 \%$ betragende Abweichung erkennen läßt. Fig. 7 
zeigt, daß die Abnahme im Prozentgehalt des $\mathrm{MgO}$ fast genau der $\mathrm{Zu}$ nahme der Brechungsexponenten proportional ist; die größte Abweichung von der Geraden bei Analyse 9 beträgt $2 \%$. Fig. 8 enthält die Prozente

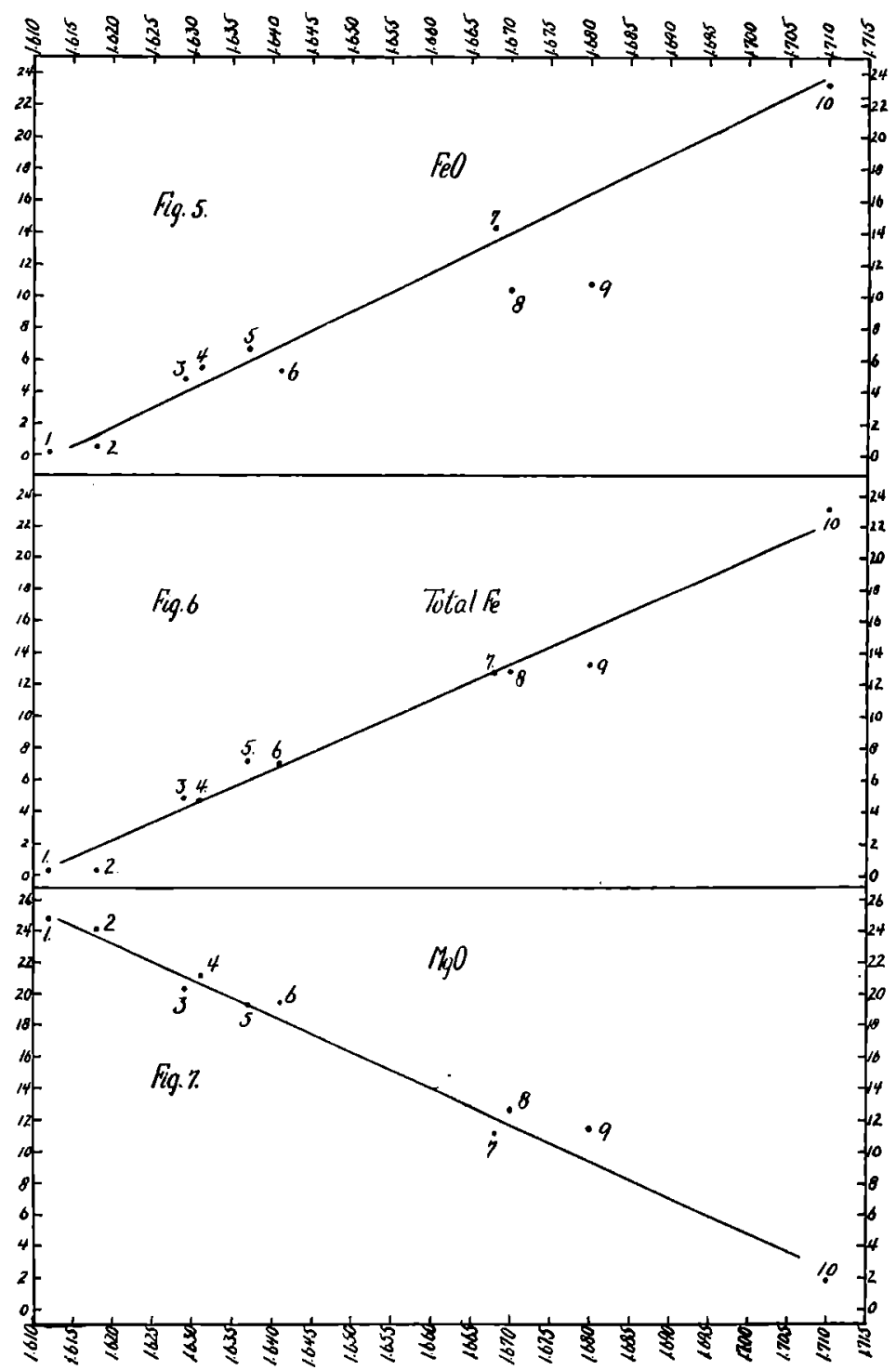

an $\mathrm{CaO}$. Bei steigendem Mittelwerte der Brechungsindices läßt sich eine geringe Abnahme von $\mathrm{CaO}$ beobachten, doch beträgt der größte Unterschied im $\mathrm{CaO}$-Gebalt überbaupt nicht viel über $3 \%$. Daher muß der Einfluß des Calciums auf die Brechungsindices in der ganzen Reihe nahezu 
der gleiche sein. Fig. 9 enthält die Prozente der Alkalien $\mathrm{K}_{2} \mathrm{O}+\mathrm{Na}_{2} \mathrm{O}$. Es läßt sich bei steigendem Mittelwert eine geringe Zunahme erkennen, da aber. der höchste Betrag der Alkalien noch unter $5 \%$ liegt, können sie nicht viel Einfluß auf die optischen Eigenschaften ausüben.

Aus dem Gesagten ist ersichtlich, daß bei dieser Serie von Amphibolen der Prozentgehalt von $\mathrm{SiO}_{2}$, Total- $\mathrm{Fe}$ und $\mathrm{Mg}$ der Änderung des Mittelwertes des Brechungsvermögens am genauesten folgt. Hauptsächlich diese Komponenten müssen daher dessen Änderung hervorrufen, und aller Wahrscheinlichkeit nach ist der Prozentgehalt des gesamten anwesenden Eisens der Faktor, der den größten Einfluß besitzt. Wenn man sich auch die Unvollständigkeit, auf deren Grundlage die obigen Figuren erhalten wurden, und den Umstand, daß man viele Ausnahmen finden wird, stets vollkommen vergegenwärtigen muß, so glaube ich doch, daß mit Hilfe dieser Figuren die chemische Zusammensetzung einer normalen und typischen Hornblende innerhalb gewisser Grenzen vorausgesagt werden kann, wenn der Mittelwert ihrer Brechungsindices bekannt ist.

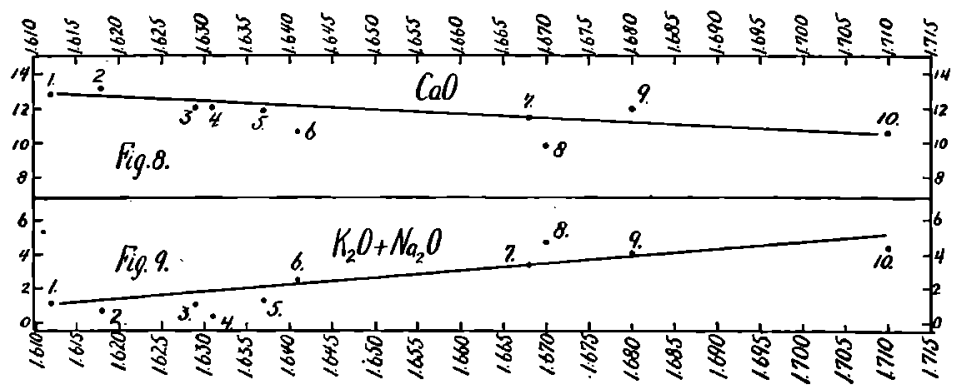

Weiterhin wird es daher von Interesse sein, zwei andere von Stanley analysierte Hornblenden zusammen mit einigen von anderen Autoren beschriebenen zu betrachten, um zu seben, wie weit sie mit den oben angegebenen übereinstimmen. Wie bereits gesagt, enthält die Literatur überraschend wenig neuere Analysen, bei denen gleichzeitig eine vollständige Beschreibung der optischen Eigenschaften gegeben wurde. In für die vorliegende Untersuchung recht ungünsliger Weise sind diejenigen Hornblenden, die überhaupt beschrieben wurden, meist ungewöhnlicher Natur, da das zu ihrer Beschreibung führende Interesse natürlich erst durch ihren außergewöhnlichen Charakter hervorgerufen wurde. Viele der Analysen kann man daher nicht als die von normalen und typischen Amphibolen betrachten. Trotzdem wird es nicht wertlos sein, sie zu untersuchen und zu versuchen, eine Erklärung für ihre Verschiedenheit von der oben angegebenen Reihe zu geben.

Analyse 11 ist ein Tremolit aus der Schweiz. Die optischen Bestimmungen gibt Kreutz an: $\alpha=1,6000 ; \beta=1,6155 ; \gamma=1,6272$; 
Mittelwert $=1,6136 . \quad \gamma-\alpha=0,0272 . \quad 2 V=85^{\circ} 30^{\prime}$ (vom Verfasser berechnet). Diese Analyse entspricht genau der Zusammensetzung, die sich aus obigen Angaben für eine Hornblende von dem angegebenen Mittelwerte des Brechungsvermögens erwarten ließ.

Analyse 12 ist die von Stanley analysierte ungewöhnliche Hornblende von Grenville, Quebec, Canada. Die optischen Eigenschaften sind folgende: $\alpha=1,6128 ; \beta=1,6180 ; \gamma=1,6328$; Mittelwert $=1,623$. $\gamma-\alpha=0,020$. $2 V=56^{\circ} 8^{\prime}$. c $\sim{ }^{\prime} c=29^{\circ} 44^{\prime}$. Für eine Hornblende mit diesem Mittelwert der Brechungsexponenten ist sie durch sehr geringen Kieselsäuregehalt, durch hohen Aluminiumgehalt, geringen Betrag der Eisenoxyde und hohen der Alkalien charakterisiert. Außerdem zeichnet sie sich speziell durch den außergewöhnlich hohen Gehalt an Fluor $(2,76 \%)$ aus. Ein anderer ebenfalls zu berücksichtigender Faktor sind die anwesenden $1,2 \% \mathrm{TiO}_{2}$. Im allgemeinen nimmt man an, $d a ß$ eine Zunahme von Fluor und Alkalien die Brechungsindices erniedrigt, und daß eine Zunahme von $\mathrm{TiO}_{2}$ gerade entgegengesetzt wirkt. Bei all diesen mit außergewöhnlichen Beträgen vertretenen Komponenten ist es schwer, zu einem brauchbaren Schluß über die Beziehung zwischen "chemischen und optischen Eigenschaften dieser Hornblende zu gelangen. Ein Axenwinkel, der so stark wie in diesem Falle von dem gewöhnlichen Werte abweicht, zeigt deullich, daß diese Hornblende von Grenville nicht als normal betrachtet werden kann.

Analyse 13 bezieht sich auf die als Pargasit bekannte Hornblende von Pargas, Finnland. Die optischen Eigenschaften sind nach Kreutz die folgenden: $\alpha=1,6158 ; \beta=1,6205 ; \gamma=1,6353 ;$ Mittelwert $=1,6255$. $\gamma-\alpha=0,0195.2 V=59^{\circ} 30^{\prime} . \quad c-c=27^{\circ} 13^{\prime}$. Wie Kreutz fand, ist dieses Mineral vollkommen analog der oben beschriebenen Hornblende von Grenville; wie diese ist sie durch einen geringen Betrag von $\mathrm{SiO}_{2}$, hohen Prozentsatz von $\mathrm{Al}_{2} \mathrm{O}_{3}$ und Alkalien und durch Anwesenheit eines ziemlich hohen Betrages von Fluor $(1,82 \%)$ charakterisiert. Die Auslöschungsschiefe und der Axenwinkel sind ebenfalls sehr ähnlich. Ebenso wie die Hornblende von Grenville kann auch diese nicht als typisch betrachtet werden.

Analyse 14 ist eine IIornblende von Chester, Massachusetts, von Duparc und Pearce beschrieben. Die optischen Eigenschaften werden wie folgt angegeben: $\alpha=1,6598 ; \beta=1,6729 ; \gamma=1,6798$; Mittelwert $=1,6698 . \quad \gamma-\alpha=0,0200 . \quad 2 V=75^{\circ} 44^{\prime}$ (vom Verfasser berechnet). $\mathfrak{c} \wedge^{\prime} c^{\prime}=14^{\circ} 40^{\prime}$. Diese Analyse stimmt ziemlich gut mit der Zusammensetzung überein, die sich für eine Hornblende von dem angegebenen Mitttelwerte des Brechungsvermögens hälte berechnen lassen. Die Beträge für $\mathrm{SiO}_{2}$ und $\mathrm{MgO}$ stimmen sehr genau mit den entsprechenden Prozenten überein, die sich aus Fig. 1 und Fig. 7 ableiten lassen. Der Betrag für die Sesquioxyde und für Tolaleisen ist etwas höher als sich hätte erwarten lassen. 
Analyse 15 bezieht sich auf eine Soretit genannte Hornblendevarietät, die von Koswinsky im nürdlichen Ural kommt und ebenfalls von Duparc und Pearce beschrieben wurde. Die optischen Eigenschaften sind die folgenden: $\alpha=1,6627 ; \beta=1,6765 ; \gamma=1,6856 ;$ Mittelwert $=1,6741$. $\gamma-\alpha=0,0228$. $2 \mathrm{~V}=82^{\circ} 30^{\prime}$ (beobachtet). $\mathfrak{c}-\frac{1}{c}=17^{\circ}$. Pleochroismus: $\mathfrak{a}=$ blaßgrünlichgelb; $\mathfrak{b}=$ grün; $\mathfrak{c}=$ tiefgrün. Die Analyse stimmt fast genau mit den aus Betrachtung von Fig. 1-9 abzuleitenden theoretischen Werten überein. Die Beträge für $\mathrm{SiO}_{2}, \mathrm{Al}_{2} \mathrm{O}_{3}$ und $\mathrm{MgO}$ weichen weniger als $2 \%$ von dem zu erwartenden Werte ab. Der Gehalt an $\mathrm{Fe}_{2} \mathrm{O}_{3}$ ist hoch und der von $\mathrm{FeO}$ entsprechend niedrig, aber der Betrag für das gesamte Eisen ist fast genau so hoch, wie sich aus Fig. 6 hätte voraussagen lassen.

Analyse 16 ist die von Stanley analysierte basaltische Hornblende von Bilin, deren optische Charaktere folgen: Mittelwert des Brechungsvermögens $=1,692$. $i$ $\_d=1^{\circ} 12^{\prime}$ im spitzen Winkel $\beta$. Pleochroismus: $\mathfrak{a}=$ lichtgelbgrün; $\mathfrak{b}=$ orange; $\mathfrak{c}=$ dunkelrötlichbraun. Absorption: $\mathfrak{c}>\mathfrak{b}>\mathfrak{a}$. Der Kieselsäuregehalt ist ungefähr der, der sich von einer Hornblende mit dem Mittelwert 1,69 erwaften läßt. Die Sesquioxyde sind niedriger, der Eisenoxydulgehalt weit geringer und der Magnesiumgehalt fast doppelt so hoch, als sich erwarten ließe. Diese Hornblende enthält $1,68 \% \mathrm{TiO}_{2}$, was zweifellos einen merklichen Einfluß auf ihre optischen Eigenschaften hat, obwohl es kaum genügend erscheint, um alle $\mathrm{Ab}-$ weichungen zu erklären. Sie ist optisch durch ihre außergewöhnliche Auslöschungsschiefe und durch die tiefrötlichbraune Farbe in der Schwingungsrichtung $\mathfrak{c}$ gekennzeichnet. Diese Hornblende zeigt zur oben untersuchten Reihe wenig Beziehungen.

Analyse 17 bezieht sich auf die als Kaersutit bekannte Hornblende von Kaersut, Grönland, wie sie von Washington beschrieben wurde. Es ist: $\alpha=1,676 ; \quad \beta=1,694 ; \gamma=1,708 ; \quad$ Mittelwert $=1,692$. $\gamma-\alpha=0,032.2 \mathrm{~V}=82^{\circ} 6^{\prime}$. Es ist keine gewöhnliche Hornblende, da sie über $10 \% \mathrm{TiO}_{2}$ enthălt. Wie sich erwarten läßt, ist daher der Betrag für das Gesamteisen zu gering für eine Hornblende mit diesen Brechungsindices. Offenbar kann man sie nicht zu den normalen Amphibolen rechnen.

Analyse 18 bezieht sich auf eine ebenfalls von Washington beschriebene ähnliche Hornblende von Linosa; ihre optischen Eigenschaften sind: $\alpha=1,692 ; \beta=1,730 ; \gamma=1,760 ;$ Mittelwert $=1,726 . \gamma-\alpha=0,068$. $2 V=79^{\circ} 54^{\prime}$. Sie enthält wie die von Kaersut einen hohen Prozentsatz $\mathrm{TiO}_{2}(8,47 \%)$. Von dieser unterscheidet sie sich durch höheren Gehalt an Eisenoxyden und geringeren Betrag der Alkalien. Optisch ist sie durch den von allen hier beschriebenen Hornblenden höchsten Mittelwert des Brechungsvermögens und durch die stärkste Doppelbrechung ausgezeichnet. 


\begin{tabular}{|c|c|c|c|c|c|c|c|c|}
\hline $\mathrm{SiO}_{2}$ & $\begin{array}{c}11 . \\
58,22\end{array}$ & $\begin{array}{c}12 . \\
45,79\end{array}$ & $\begin{array}{c}13 . \\
48,38\end{array}$ & $\begin{array}{c}14 \\
42,74\end{array}$ & $\begin{array}{c}15 . \\
40,52\end{array}$ & $\begin{array}{c}16 . \\
39,95\end{array}$ & $\begin{array}{c}17 . \\
39,52\end{array}$ & $\begin{array}{c}18 \\
40,85\end{array}$ \\
\hline $\mathrm{TiO}_{2}$ & - & 1,20 & 0,05 & 1,08 & 1,71 & 1,68 & 10,31 & 8,47 \\
\hline $\mathrm{Al}_{2} \mathrm{O}_{3}$ & 1,37 & 11,37 & 10,83 & 5,48 & 10,99 & 17,58 & 11,22 & 9,89 \\
\hline $\mathrm{F}^{2} \mathrm{e}_{2} \mathrm{O}_{3}$ & 0,04 & 0,42 & 0,76 & 11,92 & 9,64 & 7,25 & 1,22 & 8,85 \\
\hline $\mathrm{FeO}$ & 0,61 & 0,42 & 1,56 & 11,46 & 9,83 & 2,18 & 8,81 & 3,96 \\
\hline $\mathrm{MnO}$ & 0,04 & 0,39 & 0,04 & 0,06 & Sp. & Sp. & 0,06 & 0,12 \\
\hline $\mathrm{MgO}$ & 23,97 & 21,11 & 20,78 & 11,60 & 11,82 & 14,15 & 13,31 & 12,47 \\
\hline $\mathrm{CaO}$ & 12,95 & 12,71 & 12,24 & 12,72 & 12,33 & 11,96 & 10,93 & 12,16 \\
\hline$K_{2} O$ & 0,04 & 1,69 & 1,38 & 0,56 & 0,68 & 1,98 & 1,07 & 0,63 \\
\hline $\mathrm{Na}_{2} \mathrm{O}$ & 0,24 & 2,31 & 8,69 & 2,25 & 2,38 & 3,16 & 2,95 & 2,01 \\
\hline $\mathrm{H}_{2} \mathrm{O}$ & 2,17 & 0,67 & 0,91 & - & 0,50 & 0,41 & 0,59 & 0,19 \\
\hline $\boldsymbol{F}$ & 0,17 & 2,76 & 1,82 & - & - & 0,03 & - & 0,28 \\
\hline \multirow[t]{2}{*}{$\mathrm{NiO}$} & - & - & - & - & - & 0,13 & - & 0,10 \\
\hline & 99,82 & 101,06 & 101,44 & 99,69 & 100,40 & 100,46 & 100,00 & 99,98 \\
\hline \multirow[t]{2}{*}{$O=F$} & 0,07 & 1,16 & 0,76 & - & - & 0,01 & - & - \\
\hline & 99,75 & 99,90 & 100,68 & - & - & 100,45 & 一 & - \\
\hline
\end{tabular}

11. Tremolit aus der Schweiz. St. Kreutz, 'Tscherm. Mitt. (1908), 27, 251. Ausz. diese Zeitschr. (1911), 49, 280.

12. Hornblende von Grenville. S. L. Penfield und F. C. Stanley, I.c.

13. Pargasit von Pargas, Finnland. St. Kreuz, 1. c. S. 249.

14. Hornblende von Chester, Mass. L. Duparc und F. Pearce, Bull. Soc. franç. de Min. (1908), 31, 119.

15. Soretit von Koswinsky im nürdlichen Ural. L. Duparc und F. Pearce, Bull. Soc. franç. de Min. (1903), 26, 131. Ausz. diese Zeitschr. (1906), 41, 101.

16. Hornblende von Bilin. S. L. Penfield und F. C. Stanley, l. c.

17. Kaersutit von Kaersut, Grönland. H.S. Washington, Amer. Journ. of Sc. (1908), 26, 198. Ausz. diese Zeitschr. (1911), 48, 111.

18. Kaersutit von Linosa. H. S. Washington, l. c. S. 192.

Aus dem bisher Gesagten lassen sich einstweilen die folgenden Schlüsse ziehen. Für eine normale und typische Hornblende läßt sich innerhalb gewisser Grenzen die chemische Zusammensetzung aus dem Mittelwert der Brechungsexponenten voraussagen. Von den verschiedenen Oxydkomponenten schließen sich die Veränderungen im Kieselsäure-, Kalk- und Magnesiagehalt am engsten den Änderungen der Brechungsindices an. Die Tonerde schwankt innerhalb weiterer Grenzen, scheint aber auf den Brechungsindex keinen großen Einfluß zu haben. Die Eisenoxyde lassen ebenfalls, wenn auch in geringerem Maße, eine Abweichung von dem zu erwartenden Werte erkennen, auf der anderen Seite zeigt aber der Gesamteisengehalt eine genaue Übereinstimmung mit der Theorie. Durch Hinzutritt von merklichen Beträgen von Titan, Alkalien und Fluor werden deutliche Veränderungen 
der optischen Eigenschaften hervorgerufen. Die Gegenwart von Titan scheint offenbar das Brechungsvermögen zu erhöhen, während Alkalien und Fluor zu seiner Erniedrigung beitragen.

Es würde natürlich auch wertvoll sein, wenn sich mit den verfügbaren Daten, wenn möglich, eine Regelmäßigkeit in der Änderung der chemischen Zusammensetzung auffinden ließe, die in Begleitung der Änderung der Auslöschungsschiefe $\mathfrak{c} \wedge \grave{c}$, gemessen auf $b(010)$, aufträte. 16 Analysen normaler Hornblenden wurden nach dieser Richlung hin untersucht. Sie wurden nach abnehmender Auslöschungsschiefe in eine Reihe zusammengestellt. Dabei lie $B$ sich sofort erkennen, da $B$ sie sich in zwei ganz verschiedene Gruppen trennen ließen, von denen eine jede fast die gleichen Änderungen der Auslöschungsschiefe zeigte, die aber chemisch

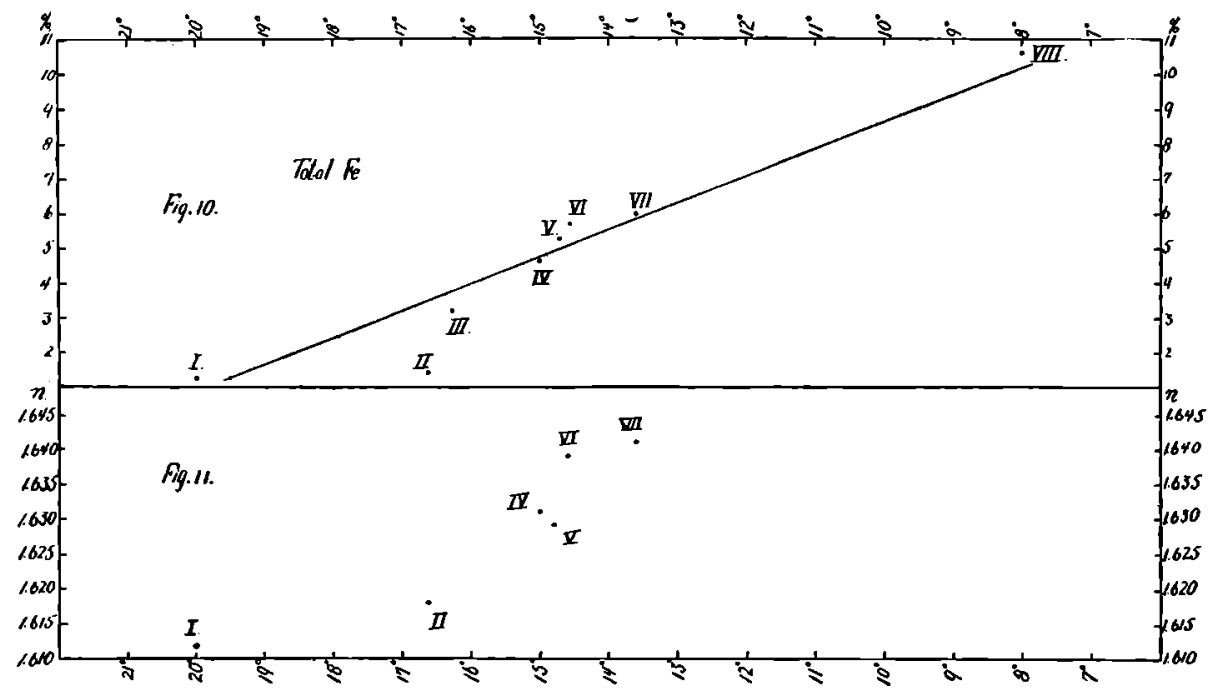

voneinander gänzlich verschieden waren. Die erste Gruppe zeigte Auslöschungsschiefen von $20^{\circ}$ bis $8^{\circ}$ bei einem Kieselsäuregehalt von etwa $58 \%$ bis $51 \%$. In der zweiten Gruppe variierte die Auslöschungsschiefe von etwa $33^{\circ}$ bis $9^{\circ}$ bei einem Kieselsäuregehalt von etwa $4 \%$ bis $36 \%$. Das heißt aber, wir hätten zweierlei Amphibole von ganz verschiedener chemischer Zusammensetzung, von denen die einen zur Tremolit-Aktinolithgruppe, die anderen zur Hornblendegruppe gehören, die aber trotzdem die gleichen Auslöschungsschiefen besitzen; man vergleiche z. B. Analyse IV und XIV auf S. 13 und 14. Diese beiden Reihen wurden infolgedessen getrennt und weiterhin einzeln betrachtet.

Die Analysen der Tremolit-Aktinolithreihe, nach abnehmender Auslöschungsschiefe geordnet, sind auf S. 13 wiedergegeben. Sie zeigen im allgemeinen bei Abnahme der Auslöschungsschiefe eine Abnahme im $\mathrm{SiO}_{2}{ }^{-}$, 
Tremolit-Aktinolithgruppe.

\begin{tabular}{|c|c|c|c|c|c|c|c|c|}
\hline $\mathrm{SiO}_{2}$ & $\begin{array}{c}1 . \\
57,47\end{array}$ & $\begin{array}{c}\text { II. } \\
57,69\end{array}$ & $\begin{array}{c}\text { III. } \\
55,02\end{array}$ & $\begin{array}{c}\text { IV. } \\
56,25\end{array}$ & $\begin{array}{c}\mathrm{V} \\
34,80\end{array}$ & $\begin{array}{c}\text { VI. } \\
55,21\end{array}$ & $\begin{array}{l}\text { VII. } \\
51,85\end{array}$ & $\begin{array}{r}\text { VIII. } \\
54,52\end{array}$ \\
\hline $\mathrm{TiO}_{2}$ & - & 0,14 & - & - & 0,10 & - & 1,26 & 0,39 \\
\hline$A l_{2} O_{3}$ & 1,28 & 1,80 & 4,53 & 1,24 & 2,58 & 3,45 & 4,36 & 9,25 \\
\hline $\mathrm{Fe}_{2} \mathrm{O}_{3}$ & 0,18 & - & 1,04 & 0,78 & 2,50 & - & 2,58 & 4,44 \\
\hline $\mathrm{FeO}$ & 0,22 & 0,55 & 3,28 & $\mathbf{B}, \mathbf{5 0}$ & 4,75 & 7,49 & 8,46 & 9,81 \\
\hline $\mathrm{MnO}$ & 0,07 & Sp. & - & 0,48 & Sp. & 一 & 0,35 & 0,46 \\
\hline $\mathrm{MgO}$ & 24,87 & 24,12 & 20,36 & 21,19 & 20,30 & 18,97 & 19,48 & 10,33 \\
\hline $\mathrm{CaO}$ & 12,84 & 13,19 & 8,00 & 12,08 & 12,08 & 10,50 & 10,60 & 1,98 \\
\hline $\mathrm{K}_{2} \mathrm{O}$ & 0,49 & 0,22 & 1,52 & 0,28 & 0,24 & - & 0,35 & 0,16 \\
\hline $\mathrm{Na}_{2} \mathrm{O}$ & 0,68 & 0,48 & 6,71 & 0,19 & 0,82 & 2,45 & 2,15 & 7,56 \\
\hline $\mathrm{H}_{2} \mathrm{O}$ & 1,30 & 1,56 & 0,51 & 1,81 & 1,60 & 1,75 & 1,21 & 1,78 \\
\hline \multirow[t]{2}{*}{$F$} & 0,77 & 0,37 & 一 & 0,04 & 0,77 & 一 & 0,46 & 一 \\
\hline & 100,19 & 100,22 & 100,97 & 99,84 & 100,65 & 99,82 & 100,24 & 100,68 \\
\hline Total Fe & 0,29 & 0,42 & 3,27 & 4,81 & 5,44 & 5,82 & 6,24 & 10,84 \\
\hline $\begin{array}{l}\text { Auslbschungs- } \\
\text { schiefe }\end{array}$ & $20^{\circ} 11$ & $16^{\circ} 38^{\prime}$ & $16^{\circ} 16^{\prime}$ & $14^{\circ} 59^{\prime}$ & $14^{\circ} 47^{\prime}$ & $14^{\circ} 94^{\prime}$ & $13^{\circ} 35^{\prime}$ & $8^{\circ}$ \\
\hline $\begin{array}{l}\text { Mittelwert } \\
\text { d. Br. }\end{array}$ & 1,612 & 1,618 & 一 & 1,631 & 4,629 & 1,639 & 1,641 & - \\
\hline
\end{tabular}

I. Richville, siehe S. 2.

II. Lee, siehe S. 2.

III. Széchenyit, siehe J. Krenner, diese Zeitschr. (1899), 31, 503.

IV. Greiner, siehe S. 3.

V. Russell, siehe S. 3.

VI. Berkeley, siehe W. C. Blasdale, Bull. Dept. Geol. Univ. Cal. (1901), 2, 333. Ausz. diese Zeitschr. (1904), 38, 689.

VII. Kragerö, siehe S. 3.

VIlI. W. C. Blasdale, l. c. S. 338 .

$\mathrm{MgO}$ - und $\mathrm{CaO}$-Gehalt und eine Zunahme im $\mathrm{Al}_{2} \mathrm{O}_{3}-, \mathrm{Fe}_{2} \mathrm{O}_{3^{-}}, \mathrm{FeO}$ - und Alkaligehalt. Diese Änderung der Zusammensetzung ist aber keineswegs regelmäßig, und die Beziehungen zwischen Zusammensetzung und Auslöschungsschiefe sind nicht ganz klar. Die einzige Verwandtschaft, die enger zu sein scheint, zeigt die Auslöschungsschiefe zu dem Betrage des Gesamteisens, dessen Zunahme die Abnahme der Auslöschungsschiefe nahezu proportional ist. Die Beziehungen zwischen diesen beiden ist in Fig. 10 graphisch dargestellt. $\mathrm{Da}$ der Alkaligehalt wenig Einfluß auf die Auslöschungsschiefe ausübt, ist aus einem Vergleich von Analyse I mit Analyse V und III mit VIII ersichtlich. Ferner muß noch erwähnt werden, daß die Abnahme der Auslöschungsschiefe von einer ziemlich stetigen Zunahme des Mittelwertes des Brechungsvermögens begleitet erscheint (vgl. Fig. 11).

Die Hornblendeanalysen sind auf S. 14, ebenfalls nach abnehmender Auslüschungsschiefe geordnet, wiedergegeben. Sie sind auf die verschiedenste 
W. E. Ford.

\section{Hornblendegruppe.}

\begin{tabular}{|c|c|c|c|c|c|c|c|c|}
\hline $\mathrm{SiO}_{2}$ & $\begin{array}{c}\text { IX. } \\
43,76\end{array}$ & $\begin{array}{c}X \\
41,99\end{array}$ & $\begin{array}{c}\text { XI. } \\
43,35\end{array}$ & $\begin{array}{c}\text { XII. } \\
40,52\end{array}$ & $\begin{array}{r}\text { XIII. } \\
45,80\end{array}$ & $\begin{array}{l}\text { XIV. } \\
39,58\end{array}$ & $\begin{array}{r}\text { XV. } \\
42,74\end{array}$ & $\begin{array}{r}\text { XVI. } \\
\mathbf{3 6 , 8 6}\end{array}$ \\
\hline $\mathrm{IiO}_{2}$ & 0,78 & 1,46 & - & 1,71 & 0,84 & Sp. & 1,08 & 1,04 \\
\hline $\mathrm{Al}_{2} \mathrm{O}_{3}$ & 8,33 & $11,6 z$ & 8,11 & 10,99 & 7,34 & 14,91 & 5,48 & 12,10 \\
\hline $\mathrm{Fe}_{2} \mathrm{O}_{3}$ & 6,90 & 2,67 & 7,91 & 9,64 & 7,55 & 4,01 & 11,92 & 7,4 \\
\hline $\mathrm{FeO}$ & 10,47 & 14,32 & 10,11 & 9,83 & 15,80 & 10,67 & 11,46 & 23,3 \\
\hline $\mathrm{MnO}$ & 0,50 & 0,25 & - & Sp. & 1,52 & Sp. & 0,06 & 0,7 \\
\hline $\mathrm{MgO}$ & 12,63 & 11,17 & 14,33 & 11,82 & 8,40 & 13,06 & 11,60 & 1,8 \\
\hline $\mathrm{CaO}$ & 9,84 & 11,52 & 13,21 & 12,33 & 12,30 & 11,76 & 12,72 & 10 \\
\hline $\mathrm{K}_{2} \mathrm{O}$ & 1,28 & 0,98 & 1,87 & 0,68 & 0,37 & 0,62 & 0,56 & $\mathbf{3}$, \\
\hline $\mathrm{Na}_{2} \mathrm{O}$ & 3,43 & 2,49 & 2,18 & 2,38 & 0,80 & 2,87 & 2,25 & $t$, \\
\hline $\mathrm{H}_{2} \mathrm{O}$ & 0,65 & 0,61 & 0,91 & 0,50 & 0,70 & 2,79 & - & 1 , \\
\hline \multirow[t]{2}{*}{$F$} & 1,82 & 0,80 & - & - & - & - & - & 0, \\
\hline & 100,49 & 99,86 & 400,98 & 100,40 & 100,82 & 100,27 & 99,69 & 99,9 \\
\hline Total $\mathrm{Fe}$ & 12,98 & 12,99 & 13,38 & 14,38 & 17,35 & 11,09 & 17,24 & 23, \\
\hline \multicolumn{9}{|c|}{ Auslöschungs- } \\
\hline $\begin{array}{l}\text { Iittelwert } \\
\text { d. Br. }\end{array}$ & 1,67 & 1,668 & - & 1,6741 & - & 一 & 1,67 & \\
\hline
\end{tabular}

IX. Renfrew, siehe S. 3.

X. Edenville, siehe S. 3.

XI. Künstliche Hornblende, K. v. Chrustschoff, N. Jahrb. f. Min. (1891), 2, 86. Ausz. diese Zeitschr. (1894), 22, 629.

XII. Soretit, L. Duparc und F. Pearce, siehe Nr.15, S.11, 1.c. S. 128. XIII. Philipstadit, R. A. Daly, Proc. Amer. Acad. of Arts and Sc. (1899), 34, 433. Ausz. diese Zeitschr. (1901), 34, 208.

XIV. Piedmont, F. R. van Horn, Amer. Geol. (1898), 21, 370. Ausz. diese Zeitschr. (1900), 32, 600.

XV. Chester, L. Duparc und F. Pearce, siehe Nr. 14, S. 11, 1. c. S. 118. XVI. Cornwall, siehe S. 3.

Weise betrachtet worden, ohne daß irgend eine brauchbare Beziehung zwischen ihrer Zusammensetzung und der Auslüschungsschiefe auffindbar gewesen wäre. Die Änderungen im Prozentgehalt der verschiedenen Oxyde sind ganz unregelmäßig. Im allgemeinen nimmt zwar der Betrag für das Gesamteisen mit abnehmender Auslöschungsschiefe zu, doch ist diese $\mathrm{Zu}$ nahme unregelmäßig (man beachte z. B. den geringen Betrag an Tolal-Fe in Analyse XIV) und zeigt auch nicht annähernd den gleichen Grad von Stetigkeit, wie es bei der Tremolit-Aktinolithgruppe der Fall ist.

Schon die Tatsache, daß es zwei Glieder der Amphibolgruppe geben kann, die bei gänzlicher Verschiedenheit der prozentualen Zusammensetzung trolzdem die gleiche Auslöschungsschiefe haben, beweist zur Genüge, daß die Grüße der Auslüschungsschiefe zum mindesten nicht direkt von der 
prozentualen chemischen Zusammensetzung abhängig ist. Wahrscheinlich muß sie letzten Endes in hohem Maße von der Art und dem Betrag der in dem Mineral vorhandenen Elemente abhängen, doch ist es wohl müglich, daß sie ebenso sehr durch die Art der Krystallstruktur, in der diese angeordnet sind, beeinflußt wird. Sicherlich sind aber die Beziehungen hier nicht einfacher Natur, und die verfügbaren Daten sind zu spärlich, um uns hoffen zu lassen, daß das Problem schon jetzt gelöst werden könnte.

Verschiedene Autoren haben schon früher versucht, eine Beziehung zwischen chemischen und optischen Eigenschaften der Amphibole zu finden. G. Murgoci hat in einer Arbeit 1), die sich hauptsächlich mit den Eigenschaften und der Klassifikation der glaukophanähnlichen Amphibole beschäftigt, auch einige allgemeine Angaben gemacht. Auf S. 362 sagt er: - Die chemische Komponente mit dem größten Einfluß auf die physikalischen Eigenschaften scheint $\mathrm{Fe}_{2} \mathrm{O}_{3}$ (bzw. $\mathrm{Fe}_{2} \mathrm{Si}_{3} \mathrm{O}_{9}$ ) zu sein, und zwar nicht nur in der Glaukophanreihe, sondern allgemein in der Hornblendefamilie... Weiterhin steht die Größe des optischen Axenwinkels, die Lage der Axenebene und selbst die Auslüschungsschiefe bis zu einem gewissen Grade in Beziehung zum Betrag des $\mathrm{Fe}_{2} \mathrm{O}_{3}$." Dies mag in bezug auf die alkalireichen, glaukophanähnlichen Hornblenden richtig sein, aber die oben geschilderten Tatsachen lassen diese Behauptung in bezug auf die gewöhnlichen Amphibole schwerlich erkennen. Der $\mathrm{FeO}$-Gehalt zeigt eine engere Verwandtschaft zu den optischen Eigenschaften als der $\mathrm{F}_{2} \mathrm{O}_{3}$-Gehalt und der Betrag für das Gesamteisen stimmt damit noch besser überein. Ferner gibt derselbe Autor auf S. 373 an: » Ein Vergleich der ganzen Gruppe der Al-Fo-Amphibole hat mich davon überzeugt, $\mathrm{da} B$ im allgemeinen die Größe der Auslöschungsschiefe weder zum $\mathrm{Al}_{2} \mathrm{O}_{3^{-}}$, noch zum $\mathrm{Fe}_{2} \mathrm{O}_{3}$-Gehalt, sondern zu dem Verhältnis ihrer Molekularkoeffizienten innerhalb der Amphibolkonstilution in Beziehung steht.\& Die Betrachtung der oben wiedergegebenen Analysen hat diese Theorie nicht bestätigen können. Es genügt tatsächlich schon ein flüchtiger Blick auf die Prozente an $\mathrm{Al}_{2} \mathrm{O}_{3}$ und $\mathrm{Fe}_{2} \mathrm{O}_{3}$ in den auf S. 13 und 14 zitierten Analysen, um zu erkennen, daß diese Annahme bei ihnen nicht erfüllt ist.

Die Tatsache, daß der Eisengehalt einer Hornblende großen Einfluß auf ihre optischen Eigenschaften hat, ist schon 1871 von Tschermak ${ }^{2}$ ) erwähnt und neuerdings wieder von Kreu(z ${ }^{3}$ ) betont worden. Wie bereits angegeben, gelangten wir mit den Resultaten dieser Arbeit zu der gleichen Erkenntnis.

1) >Beiträge zur Klassifikation der Amphibolec. Bull. of the Geol. Dept. Univ. of Calif (1906), 4, 359, Nr. 15. Ausz. diese Zeitschr. (1909), 46, 391.

2) G. Tschermak, Min. Mitt. (1871), S. 17.

3) St. Kreutz, Tscherm. Mitt. (1908), 27, 250. 
Wiik $\left.{ }^{1}\right)$ versuchte zu zeigen, daß die Größe der Auslöschungsschiefe, $c \sim \stackrel{1}{c}$, proportional dem $\mathrm{Al}_{2} \mathrm{O}_{3}$-Gehalt zunähme. Diese Theorie wurde schon von anderen als unbaltbar erklärt; die Ergebnisse dieser Arbeit fübren uns zu dem gleichen Schluß.

Mineralogisches Laboratorium der Sheffield Scientific School, Yale Universität, New Haven, Conn, 2. Juli 1913.

1) F. J. Wiik, diese Zeitschr. (1883), 7, 79. 